\title{
Positively charged micelles based on a triblock copolymer demonstrate enhanced corneal penetration
}

\author{
Jingguo $\mathrm{Li}$ \\ Zhanrong Li \\ Tianyang Zhou \\ Junjie Zhang \\ Huiyun Xia \\ Heng Li \\ Jijun $\mathrm{He}$ \\ Siyu $\mathrm{He}$ \\ Liya Wang
}

Henan Eye Institute, Henan Eye Hospital, Henan Provincial People's Hospital and Zhengzhou University

People's Hospital, Zhengzhou,

People's Republic of China
Correspondence: Junjie Zhang Henan Eye Institute, Henan Eye Hospital, Henan Provincial People's Hospital and Zhengzhou University People's Hospital, Number 7 Weiwu Road, Zhengzhou 450003, People's Republic of China

Tel +8637165580919

Fax +86 37I 65952907

Email zhangji66@126.com
This article was published in the following Dove Press journal:

International Journal of Nanomedicine

28 September 2015

Number of times this article has been viewed

Purpose: The cornea is a main barrier to drug penetration after topical application. The aim of this study was to evaluate the abilities of micelles generated from a positively charged triblock copolymer to penetrate the cornea after topical application.

Methods: The triblock copolymer poly(ethylene glycol)-poly( $\varepsilon$-caprolactone)-gpolyethyleneimine was synthesized, and the physicochemical properties of the self-assembled polymeric micelles were investigated, including hydrodynamic size, zeta potential, morphology, drug-loading content, drug-loading efficiency, and in vitro drug release. Using fluorescein diacetate as a model drug, the penetration capabilities of the polymeric micelles were monitored in vivo using a two-photon scanning fluorescence microscopy on murine corneas after topical application.

Results: The polymer was successfully synthesized and confirmed using nuclear magnetic resonance and Fourier transform infrared. The polymeric micelles had an average particle size of $28 \mathrm{~nm}$, a zeta potential of approximately $+12 \mathrm{mV}$, and a spherical morphology. The drug-loading efficiency and drug-loading content were $75.37 \%$ and $3.47 \%$, respectively, which indicates that the polymeric micelles possess a high drug-loading capacity. The polymeric micelles also exhibited controlled-release behavior in vitro. Compared to the control, the positively charged polymeric micelles significantly penetrated through the cornea.

Conclusion: Positively charged micelles generated from a triblock copolymer are a promising vehicle for the topical delivery of hydrophobic agents in ocular applications.

Keywords: corneal barriers, polymeric micelles, topical administration, corneal penetration, controlled release

\section{Introduction}

Due to their ease of use, low invasiveness, and simple formulation, topically applied drugs are preferred for ophthalmic drug administration, especially to the cornea, the ocular surface, and the anterior segment. ${ }^{1,2}$ However, the unique structure of the cornea with respect to its specific physiological, anatomical, and biochemical features restricts the penetration of drugs into target sites. ${ }^{3,4}$ Furthermore, the eye possesses specific defense mechanisms, such as lacrimation, tear dilution, and tear turnover, which can reduce the residential time of a drug solution to 5-6 minutes in the precorneal space after topical administration, with only $1 \%-3 \%$ of the solution ultimately penetrating into the cornea and reaching the intraocular tissues. ${ }^{5,6}$

This low penetration after topical application is primarily because drug entry into the ocular globe is restricted predominantly by corneal barrier functions, which serve to protect the anterior ocular segment, and by the conjunctival-scleral barrier, which 
serves to protect the intermediate and posterior segments. ${ }^{7,8}$ The anterior corneal barrier is composed of epithelial tight junctions and possesses unique physicochemical properties that involves the opposite characteristics of lipophilic epithelium and hydrophilic stroma. ${ }^{9}$ The epithelium and the stroma present different physicochemical properties, and the movement of hydrophilic compounds is limited to the epithelium, whereas the movement of lipophilic compounds is limited to the stroma. The dual nature of the cornea acts as a strong barrier to drugs, which protects the ocular globe. ${ }^{10,11}$ Therefore, enhancing the ocular penetration of eye drops remains one of the most challenging goals of ophthalmology. ${ }^{12}$

Over the past few decades, the use of polymeric nanomicelles that can self-assemble from amphiphilic block copolymers as delivery vehicles for the controlled delivery and solubilization of water-insoluble drugs has been the focus of increasing interest. ${ }^{13-15}$ Polymeric micelles possess many excellent properties as drug-delivery systems, including high kinetic and thermodynamic stability, suitable size (20-100 nm), narrow size distribution, easily adjustable surface potential, modulated biocompatibility, tissue accumulation and penetration, controlled intracellular trafficking, controlled release, and reduced inherent toxicity. ${ }^{13,16}$ Recently, polymeric micelles have been used for ocular drug delivery to improve the drug release. ${ }^{17,18}$ Drug-loaded polymeric micelles offer favorable biological properties, such as biocompatibility, biodegradability, mucoadhesiveness, nontoxicity, and controlled drug release. ${ }^{19}$ Topical formulations of drug-loaded polymeric micelles hold significant promise for ophthalmic drug delivery. ${ }^{20,21}$

The particle size and potential of polymeric micelles are the two main factors that influence their ophthalmic application. ${ }^{22}$ Small, positively charged nanoparticles may be useful as penetration enhancers for ocular drug delivery. The small size of nanomicelles enables them to pass through the corneal barrier with ease. Polymeric micelles with positive surface charges possess mucoadhesive properties based on the electrostatic interactions that occur between their positively charged groups and the negatively charged mucin sialic acid residues that exist on the ocular surface. These nonspecific electrostatic interactions facilitate cell uptake of the polymeric micelles through negatively charged cell membranes. Therefore, polymeric micelles with positive surface charges have prolonged residence time on the ocular surface and can enhance the corneal penetration of polymeric micelle-loaded drugs.

In the present study, the triblock copolymer poly(ethylene glycol)-poly( $\varepsilon$-caprolactone)-g-polyethyleneimine
(PEG-PCL-g-PEI), denoted as PCI, was synthesized and characterized. The block copolymer could self-assemble into polymeric micelles with positive potential, and the physicochemical properties of the self-assembled micelles were investigated. First, we examined the in vitro drug-release behavior of the drug-loaded micelles. Then, we used the hydrophobic dye fluorescein diacetate (FDA) as a model drug to examine their corneal penetration in vivo in C57BL/6 mice using two-photon laser microscopy.

\section{Materials and methods Materials}

$\varepsilon$-Caprolactone ( $\varepsilon$-CL) (from Sigma-Aldrich, St Louis, MO, USA) was purified by vacuum distillation over $\mathrm{CaH}_{2}$. $\alpha$-Methoxy-3-hydroxy-poly(ethylene glycol) (mPEG-OH, $\mathrm{Mn}=2 \mathrm{kDa}$ ), polyethyleneimine (PEI, molecular weight $[\mathrm{Mw}]=1,800 \mathrm{Da})$, and stannous octoate $\left(\mathrm{Sn}(\mathrm{Oct})_{2}\right)$ were purchased from Sigma-Aldrich and used as received. Maleic anhydride, dicyclohexylcarbodiimide, $N$-hydroxysuccinimide, and FDA were all from Aladdin (Shanghai, People's Republic of China) and were used as received. Cyclosporine (CyA) was obtained from Zhejiang Ruibang Laboratories (Wenzhou, People's Republic of China), and used as received. Dialysis bags (Mw cut-off: $1 \mathrm{kDa}, 3.5 \mathrm{kDa}$, $14 \mathrm{kDa}$ ) were purchased from Shanghai Green Bird Technology Development Co., Ltd. (Shanghai, People's Republic of China, and stored in $1 \mathrm{mM}$ ethylene diaminetetraacetic acid aqueous solution prior to use. Chloroform was dried over $\mathrm{CaH}_{2}$ and then distilled under ambient pressure. All other reagents were of analytical grade and used as received.

C57BL/6 mice, which were free of clinically observable ocular surface disease and were of specific pathogen-free grades, were purchased from Beijing Vital River Laboratory Animal Technology Co., Ltd. (Beijing, People's Republic of China). All the mice used were of age 4 months. All animal care and experimental protocols were approved by the Ethical Committee of Experimental Animal Care of Henan Eye Institute and complied with the National Institutes of Health guidelines, and all procedures in the study conformed to the Association for Research in Vision and Ophthalmology Statement for the Use of Animals in Ophthalmic and Vision Research.

\section{Synthesis and characterization of mPEG- PCL-g-PEl triblock copolymer}

First, a predetermined amount of $\mathrm{mPEG}$-PCL was synthesized via ring-opening polymerization of $\varepsilon-\mathrm{CL}$, which was initiated by $\mathrm{mPEG}-\mathrm{OH}$ and catalyzed by $\mathrm{Sn}(\mathrm{Oct})_{2}$ according 
to the literature. ${ }^{23}$ Briefly, mPEG and $\varepsilon$-CL (at an amount calculated to result in the designated PCL block length) were placed into a reaction flask and dried at reduced pressure at a temperature of $70^{\circ} \mathrm{C}$ for 2 hours. Subsequently, the flask was purged with dry argon and $\mathrm{Sn}(\mathrm{Oct})_{2}(0.1 \mathrm{~mol} \%$ corresponding to $\varepsilon-C L)$, sealed under dry argon, and immersed in an oil bath. The bulk polymerization was performed at a temperature of $115^{\circ} \mathrm{C}$ for 24 hours. The crude polymer mPEG-PCL-OH was purified by precipitating twice into cold diethyl ether from a tetrahydrofuran solution and was then vacuum dried at $40^{\circ} \mathrm{C}$. The degree of polymerization of the PCL block was calculated by comparing the integrals of the characteristic peaks in ${ }^{1} \mathrm{H}$ NMR (nuclear magnetic resonance) spectra of the PCL block at $\sim 2.25 \mathrm{ppm}$ (triplet, $-\mathrm{C}(=\mathrm{O})-\mathrm{CH}_{2}-$ ) and the PEG block at $3.36 \mathrm{ppm}$ (singlet, $-\mathrm{OCH}_{3}$ ). The yield of mPEG-PCL-OH was $>96 \%$.

In a second reaction step, a monocarboxy-capped diblock copolymer (mPEG-PCL-COOH) was obtained via hydroxyl esterification of mPEG-PCL-OH with succinic anhydride, according to a previously reported method. ${ }^{24}$ Typically, the monohydroxy-terminated diblock copolymer (1 mmol) and maleic anhydride $(10 \mathrm{mmol})$ were dissolved in dry chloroform $(100 \mathrm{~mL})$ and placed in a two-necked flask equipped with a magnetic stirring bar and a reflux condenser. The mixture was stirred under a dry argon atmosphere at $70^{\circ} \mathrm{C}$ for 72 hours. The mixture was filtered, and crude polymer was obtained by precipitation into cold diethyl ether and redissolved in dichloromethane. The dichloromethane solution was washed three times with aqueous hydrochloric acid $(10 \% \mathrm{v} / \mathrm{v})$ and then four times with a saturated $\mathrm{NaCl}$ solution. Then, the organic phase was isolated, dried over magnesium sulfate, and filtered. The copolymer was recovered by precipitation into cold diethyl ether and vacuum dried to a constant weight at $50^{\circ} \mathrm{C}$ to eliminate any water and maleic anhydride residues. The yield of $\mathrm{mPEG}-\mathrm{PCL}-\mathrm{COOH}$ was $>90 \%$.

Finally, in a third reaction step, the block copolymer mPEG-PCL-g-PEI was synthesized according to our previously reported method. ${ }^{25}$ A predetermined amount of $\mathrm{mPEG}-$ PCL-COOH and $\mathrm{N}$-hydroxy-succinimide was dissolved in $20 \mathrm{~mL}$ of chloroform and placed into a flask equipped with a magnetic stir bar. The flask was cooled in an ice-water bath, and dicyclohexylcarbodiimide was added; the flask was then sealed under argon. The reaction mixture was stirred at $0^{\circ} \mathrm{C}$ for 1 hour, and then the solution of PEI in chloroform was slowly added. The reaction mixture was stirred at $0^{\circ} \mathrm{C}$ for 1 hour and then at room temperature for an additional 24 hours. The precipitated dicyclohexylurea was removed by filtration, and the filtrate was added to excessively cold diethyl ether. The copolymer was collected by filtration and dried under vacuum at room temperature. The chemical composition and $\mathrm{Mw}$ were confirmed by ${ }^{1} \mathrm{H}$ NMR spectra. The yield of mPEG-PCL- $g$ PEI was $>85 \%$. The yields of all three steps are reported by the means of $\mathrm{mPEG}_{2000}-\mathrm{PCL}_{2500}-g-\mathrm{PEI}_{1800}$.

\section{Characterization}

${ }^{1} \mathrm{H}$ NMR spectra were obtained using a Varian Unity $300 \mathrm{MHz}$ spectrometer. Either chloroform- $d_{1}$ or methanol- $d_{4}$ was used as a solvent, depending on polymer solubility. Fourier transform infrared spectroscopy (FTIR) spectral studies were performed using a Nicolet/Nexus 670 FTIR spectrometer in a range between $4,000 \mathrm{~cm}^{-1}$ and $500 \mathrm{~cm}^{-1}$ with a resolution of $2 \mathrm{~cm}^{-1}$. All powder samples were compressed into $\mathrm{KBr}$ pellets prior to FTIR measurements. The Mws and Mw distribution of the polymers were estimated using a gel permeation chromatography (GPC) system consisting of a styroldivinylbenzole-copolymer linear column (Polymer Standard Service, Mainz, Germany) with dichloromethane as an eluent ( $1 \mathrm{~mL} / \mathrm{min})$; polystyrene was used as a standard for calibration. Samples for scanning electron microscopy (SEM, Quanta 400F) analysis were prepared by drying a dispersion of the micelle solution on a clean glass slide, which was first washed with deionized water and then with acetone for 24 hours in a desiccator. A thin layer of gold was sputter coated on the samples for charge dissipation during SEM imaging.

\section{Preparation of FDA-loaded micelles}

FDA-loaded micelles were prepared using a solvent evaporation method as follows: the triblock copolymer PCI (10 mg) and FDA $(0.5 \mathrm{mg})$ were dissolved in $2 \mathrm{~mL}$ of methanol/ acetonitrile (1:1) in a glass vial. Then, the solution was added dropwise into $20 \mathrm{~mL}$ of phosphate buffered saline under ultrasonic agitation using a Type 60 Sonic Dismembrator (Thermo Fisher Scientific, Waltham, MA, USA). The organic solvent was then completely removed by vacuum distillation using a rotary evaporator to allow micelle formation. The micelle solution was concentrated and washed three times using a MILLIPORE Centrifugal Filter Device (Mw cut off: $100,000 \mathrm{Da})$ to remove the free FDA dissolved in the micelle solution. It was then filtered through a syringe filter (pore size: $0.45 \mu \mathrm{m}$ ) to eliminate large polymer or FDA aggregates. The filtered FDA-loaded micelles were mixed with a sterile solution of $\mathrm{NaCl}$ in water $(9 \%, \mathrm{w} / \mathrm{w})$ at a volume ratio of $9: 1$. Finally, the $\mathrm{NaCl}-$ water solution $(0.9 \%$, w/w) containing the FDA micelles was prepared as eye drops. CyA-loaded micelles and blank micelles were prepared without varying 
the preparation conditions or procedure. FDA nanoparticles and FDA microparticles were prepared by a reprecipitation method that has been described in the literature. ${ }^{9}$

\section{Micelle particle size and zeta potential}

The hydrodynamic sizes, polydispersity indexes (PDIs), and zeta potentials of the micelles were determined via dynamic light scattering. The micelles were mixed into standard buffered saline and filtered through a $450 \mathrm{~nm}$ syringe filter. Measurements were performed at $25^{\circ} \mathrm{C}$ using a 90 Plus/ BI-MAS device (Brookhaven Instruments Corporation, New York, NY, USA). For the $\zeta$-potential measurements, a standard electrophoresis minicell from Brookhaven was used. The data for particle size and $\zeta$-potential were collected on an autocorrelator with scattered light detection angles at $90^{\circ}$ and $15^{\circ}$, respectively. For each sample, the data from five measurements were averaged to obtain the mean particle size and $\zeta$-potential.

\section{Determination of drug-loaded contents}

The CyA-loaded content was defined as the weight percentage of drug in the micelle. To determine the drug-loading content (DLC), preweighed, freeze-dried micelles were redissolved in acetonitrile/methanol (1/1 volume ratio). High-performance liquid chromatography analysis was conducted on a Waters 2,695 system (Waters, Milford, MA, USA) equipped with an X-BridgeTM C18 column $(3.5 \mu \mathrm{m}, 3.0 \mathrm{~mm} \times 150 \mathrm{~mm}$, Waters) and UV/vis detector. The flow rate was $0.8 \mathrm{~mL} /$ $\mathrm{min}$, and the detection wavelength was $225 \mathrm{~nm}$. The column temperature was $50^{\circ} \mathrm{C}$, and the sample injection volume was $20 \mu \mathrm{L}$. The quantity of CyA was calculated based on a calibration curve of CyA in acetonitrile/water (79/21 volume ratio). The DLC ( $w \mathrm{t} \%$ ) and drug-loading efficiency (DLE, $\mathrm{wt} \%$ ) were calculated according to the following formulas:

$$
\begin{gathered}
\text { DLC }=\left(\frac{\text { Amount of loaded drug }}{\text { Amount of drug-loaded micelle }}\right) \times 100 \% \\
\text { DLE }=\left(\frac{\text { Amount of loaded drug }}{\text { Amount of feeding drug }}\right) \times 100 \%
\end{gathered}
$$

\section{In vitro drug release}

The in vitro drug-release behavior of the drug-loaded micelles was investigated using a previously published bulk equilibrium reverse dialysis bag technique. ${ }^{26}$ Briefly, a preprepared CyA-loaded PCI micelle solution in phosphate/citrate buffer in phosphate buffered saline $(\mathrm{pH}$ 7.2) was directly placed into a $200 \mathrm{~mL}$ stirring sink solution in which numerous dialysis sacs containing $1 \mathrm{~mL}$ of the same sink solution were previously immersed. These sacs were equilibrated with the sink solution for $\sim 30$ minutes prior to experimentation. The release study was performed at $35^{\circ} \mathrm{C}$ in an incubator shaker (Shanghai Yiheng Scientific DKZ, Shanghai, People's Republic of China). At predetermined time intervals, the dialysis bags were withdrawn, and the contents were assayed by high-performance liquid chromatography analysis. The cumulative amount of released drug was calculated based on a calibration curve of CyA in acetonitrile/water (79/21 volume ratio), and the percentage of drug release from micelles was plotted against time. The release experiments were conducted in triplicate.

\section{Animal preparation and in vivo three- dimensional imaging}

The mice were anesthetized via intraperitoneal injections of pentobarbital sodium ( $85 \mathrm{mg} / \mathrm{kg}$ body weight) (SigmaAldrich). A homemade, plastic eyecup was mounted on the upward-facing eye and was sealed with Vaseline (Zhengzhou Paini Chemical Reagent Factory, Zhengzou, People's Republic of China). Next, $1 \mathrm{mg} / \mathrm{mL}$ of the FDA-loaded micelle solution was instilled into the eyecup to enable clear imaging of the corneal layers. The dye solution remained on the eye for 30 minutes and was then removed; the cornea was then rinsed three times with $0.9 \%$ saline water. Then, the mice were placed on a custom-made plastic plate with the treated cornea facing up. Movement of the head and four limbs during the imaging session was limited by gentle physical restraint with adhesive tape. The eyecup was filled with a $0.9 \%$ saline solution to bridge the working distance of the water immersion objective and to minimize heartbeat- and breathing-induced eyeball movement. In vivo two-photon three-dimensional (3-D) imaging was immediately performed.

The two-photon imaging was performed using a Zeiss LSM 780 NLO fluorescence microscope system (Carl Zeiss Meditec AG, Jena, Germany) with Zeiss control software (Zen 2010; Carl Zeiss Meditec AG). This system is a combination of two-photon excitation and laser scanning confocal microscope based on an upright Axio Examiner microscopy. In addition to its regular laser scanning confocal capabilities with 32-channel GaAsP spectral detectors, this system has a tunable, mode-locked $2 \mathrm{PH}$ Ti-sapphire laser (Chameleon Vision II; Coherent, Inc., Santa Clara, CA, USA) with an excitation range of $780 \mathrm{~nm}$ and three reflected light nondescanned detectors.

All the imaging were performed using a $20 \times$ (Plan-Apochromat, NA =1.0; Carl Zeiss Meditec AG) waterimmersion objective. Two-photon imaging signals were 
separately detected using two reflected light nondescanned detectors. The laser power used in this study was between $24 \%$ and $28 \%$ of maximum laser power $(>3.5 \mathrm{~W})$. The pinhole was set to the maximal open setting for this application, and the samples were scanned using a $2 \mathrm{~mm} z$-axis step size to generate 3-D data sets extending from the corneal endothelium to the epithelial surface. The image sequences were recorded as 12-bit, 512×512-pixel images. The scanning rate used in our study was $968.14 \mathrm{~ms}$ per frame. Corneal 3-D data were collected from the central to the paracentral region.

\section{Results}

\section{Synthesis and characterization of block copolymers}

The triblock copolymer PCI was synthesized via a combination of ring-opening polymerization of $\varepsilon-\mathrm{CL}$ and an amidation reaction between the amine group of PEI and the carboxyl group of PCL, as outlined in Figure 1. The chemical structures of the copolymers were confirmed by ${ }^{1} \mathrm{H}$ NMR and FTIR spectroscopy. In Figure 2, the ${ }^{1} \mathrm{H}$ NMR spectra of the triblock copolymer PCI and the propolymer clearly show characteristic resonance peaks of PEG, PCL, and PEI. The sharp peak appearing at $3.64 \mathrm{ppm}$ was attributed to the methylene protons in the PEG blocks. The typical signals of the methylene protons in the PCL blocks appeared at $1.32 \mathrm{ppm}, 1.56 \mathrm{ppm}, 2.31 \mathrm{ppm}$, and $4.06 \mathrm{ppm}$. The strong and broad absorption peaks that were observed at 2.5-2.9 ppm were assigned to the methane protons of PEI. ${ }^{27}$ The ${ }^{1} \mathrm{H}$ NMR results showed that the PCI copolymer was successfully synthesized.

Figure 3 shows the FTIR spectra of the diblock intermediates and final triblock PCI copolymer. The PEG and PCL blocks were characterized by prominent absorptions at $\sim 1,190 \mathrm{~cm}^{-1}\left(\mathrm{~s}, v_{\mathrm{C}-0}\right)$ and $1,726 \mathrm{~cm}^{-1}\left(\mathrm{~s}, v_{\mathrm{C}=0}\right)$, respectively. Obviously, both PEG and PCL blocks are present in both the intermediate prepolymers and the final copolymer. The two new and strong absorption peaks that appeared in the spectrum of the triblock copolymer at $\sim 1,644 \mathrm{~cm}^{-1}$ and $1,562 \mathrm{~cm}^{-1}$ were attributed to amide linkages $\left(\mathrm{s}, v_{\text {amide, } \mathrm{C}=\mathrm{O}}\right)$. In addition, the PCI copolymer showed a strong and broad characteristic absorption of amines at $\sim 3,300 \mathrm{~cm}^{-1}\left(\mathrm{~s}, v_{\mathrm{N}-\mathrm{H}}\right)$.

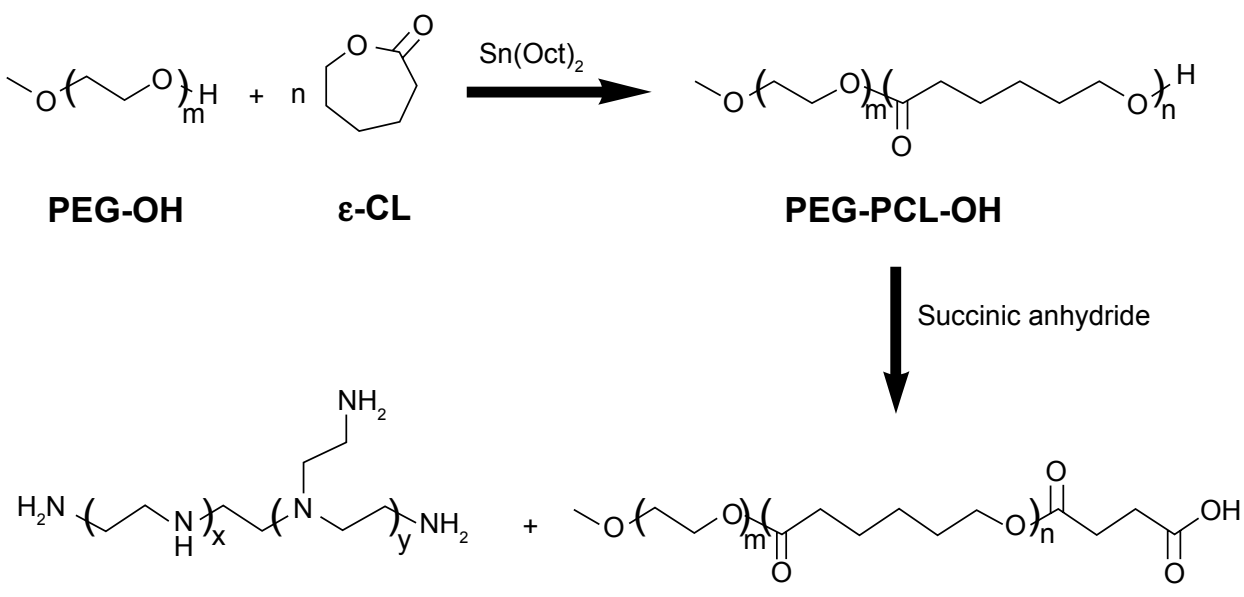

PEI PEG-PCL-COOH

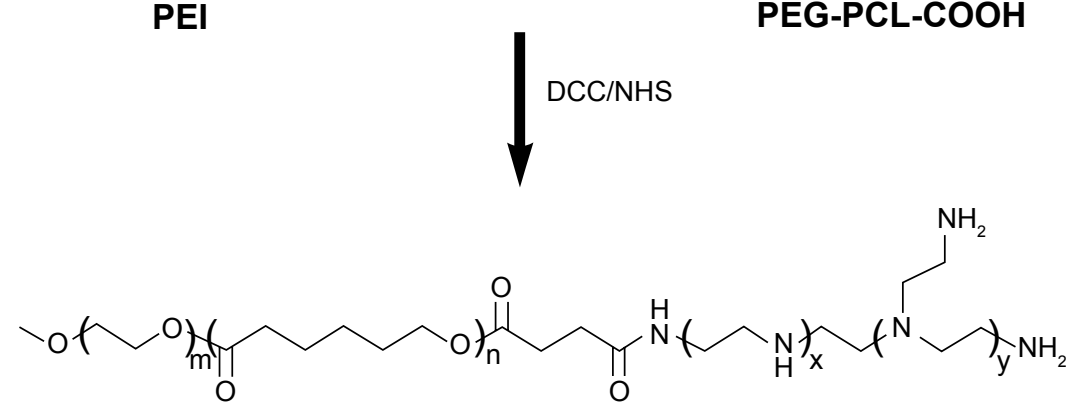

\section{PEG-PCL-g-PEI}

Figure I Synthetic route of the copolymer.

Abbreviations: $\varepsilon-C L$, $\varepsilon$-caprolactone; PEI, polyethyleneimine; DCC, dicyclohexylcarbodiimide; NHS, N-hydroxy-succinimide; PEG-PCL-g-PEI, poly(ethylene glycol)-poly ( $\varepsilon$-caprolactone)-g-polyethyleneimine. 


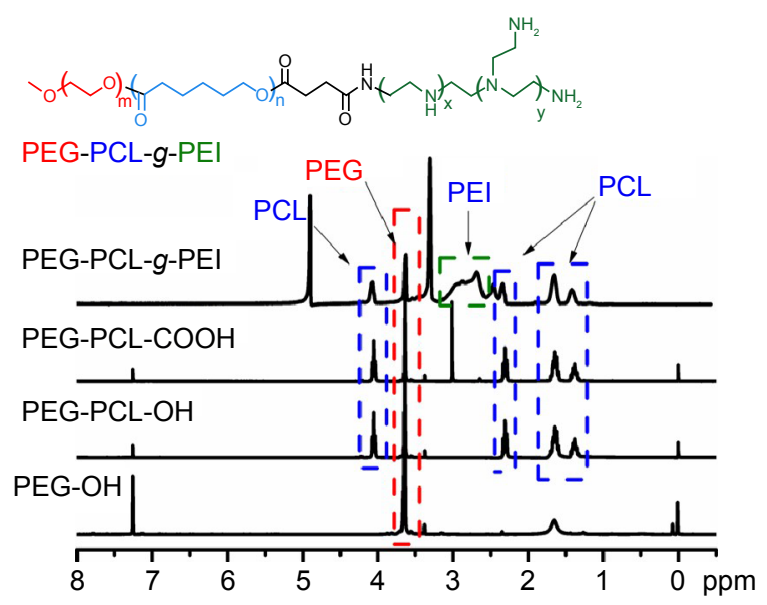

Figure 2 'H NMR spectra of PEG-OH, PEG-PCL-OH, PEG-PCL-COOH in chloroform- $d_{1}$ and PEG-PCL-g-PEI in methanol- $d_{4}$ recorded on a Varian Unity $300 \mathrm{MHz}$ spectrometer.

Abbreviations: NMR, nuclear magnetic resonance; PEG, poly(ethylene glycol); $\mathrm{PCL}$, poly( $\varepsilon$-caprolactone); $\mathrm{PEI}$, polyethyleneimine.

The FTIR results are consistent with the expected structures of the intermediate prepolymers and the final copolymers.

The block components of the copolymers were calculated using the integral intensities of the peaks in the ${ }^{1} \mathrm{H}$ NMR spectra as previously reported. ${ }^{24}$ The results indicated that the block copolymers had Mws of 2,000 Da, 2,500 Da, and $1,800 \mathrm{Da}$, which are consistent with the requirements of the original design. Furthermore, GPC measurements were utilized to determine the average $\mathrm{Mw}$ and polydispersity. The number average $\mathrm{Mw}(\mathrm{Mn})$ and polydispersity were determined to be $6,073 \mathrm{Da}$ and 1.74 , respectively. The Mws of the copolymers that were estimated by the GPC measurements were close to those that were calculated from the ${ }^{1} \mathrm{H}$ NMR spectra.

\section{Preparation and characterization of $\mathrm{PCl}$ micelles}

As an amphiphilic block copolymer, mPEG-PCL-g-PEI can self-assemble into polymeric micelles in an aqueous phase with

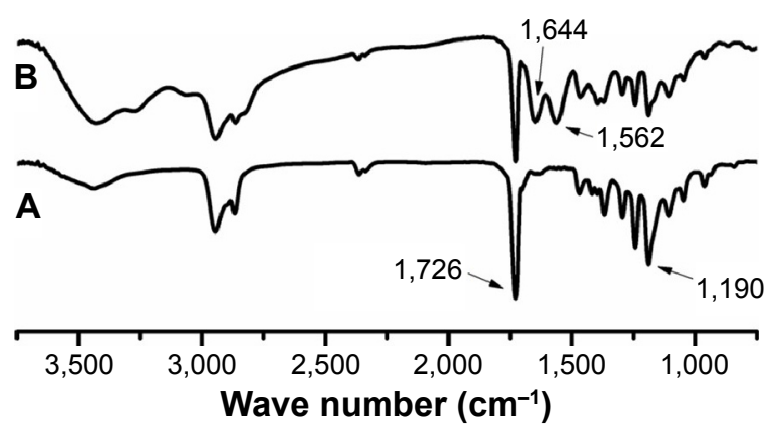

Figure 3 FTIR spectra of the diblock intermediates PEG-PCL-COOH (A) and PCl (B). Abbreviations: $\mathrm{PEG}$, poly(ethylene glycol); $\mathrm{PCL}$, poly( $\varepsilon$-caprolactone); $\mathrm{PCl}$, poly (ethylene glycol)-poly(E-caprolactone)-g-polyethyleneimine; FTIR, Fourier transform infrared spectroscopy.
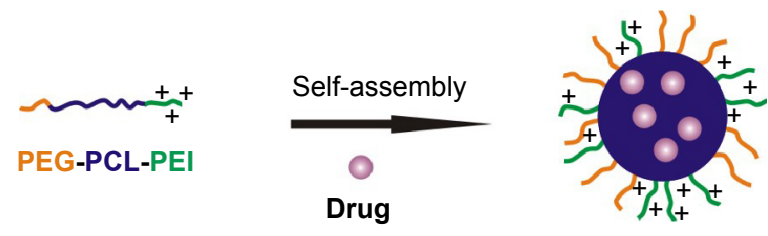

Drug-loading micelle

Figure 4 Schematic illustration of the formation of drug-loaded polymeric micelles.

Abbreviations: PEG, poly(ethylene glycol); PCL, poly(E-caprolactone); PEI, polyethyleneimine.

a positive charge. ${ }^{28}$ These cationic nanomicelles can be loaded with hydrophobic drugs; a schematic illustration is shown in Figure 4. The sizes and zeta potentials of the polymeric micelles were determined via dynamic light scattering, and the results are shown in Table 1 . The micelles had a relatively small average hydrodynamic diameter of $27.74 \mathrm{~nm}$, a PDI of 0.212 , and a moderate positive charge of $12.12 \mathrm{mV}$. The morphology of the polymeric micelles was determined by SEM and appeared to be uniform and spherical in shape (Figure 5A).

\section{Drug loading and in vitro drug-release studies}

It was found that the DLC of the micelles increased with an increasing feed ratio of drug to PCI during micelle preparation, whereas the DLE decreased (Table 2). Although a sufficient DLC (eg, 8.32\%) was achieved at a high feed ratio, the DLE was very low (13.6\%). In this study, a DLC of $3.47 \%$ and a DLE of $75.37 \%$ in a $1: 20$ drug polymer ratio was adopted.

As shown in Figure 5B, a typical biphasic-release profile was observed, with an initial rapid release followed by a sustained slow release over a prolonged time of up to several hours. The drug release from the polymeric micelles with a DLC of $3.47 \%$ reached $44.8 \%$ in 12 hours. The burst release was $31.7 \%$ after 2 hours of drug release.

\section{In vivo studies}

The cornea is a transparent tissue; therefore, the morphological and structural features of entire corneas could be acquired

Table I Average particle sizes and zeta potentials of polymeric micelles and nanoparticles

\begin{tabular}{llll}
\hline & $\begin{array}{l}\text { Size } \\
(\mathbf{n m})\end{array}$ & Polydispersity & $\begin{array}{l}\text { Zeta } \\
\text { potentials }(\mathbf{m V})\end{array}$ \\
\hline PCI micelle & 27.74 & 0.212 & +12.12 \\
FDA nanoparticle & 214.3 & 1.000 & -0.80 \\
FDA macroparticle & $\mathrm{I}, 402$ & 0.587 & $-1.1 \mathrm{I}$ \\
PEG-PCL micelle & 36.32 & 0.152 & -0.24 \\
\hline
\end{tabular}

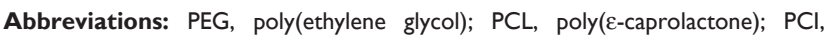
poly(ethylene glycol)-poly( $\varepsilon$-caprolactone)-g-polyethyleneimine; FDA, fluorescein diacetate. 
A

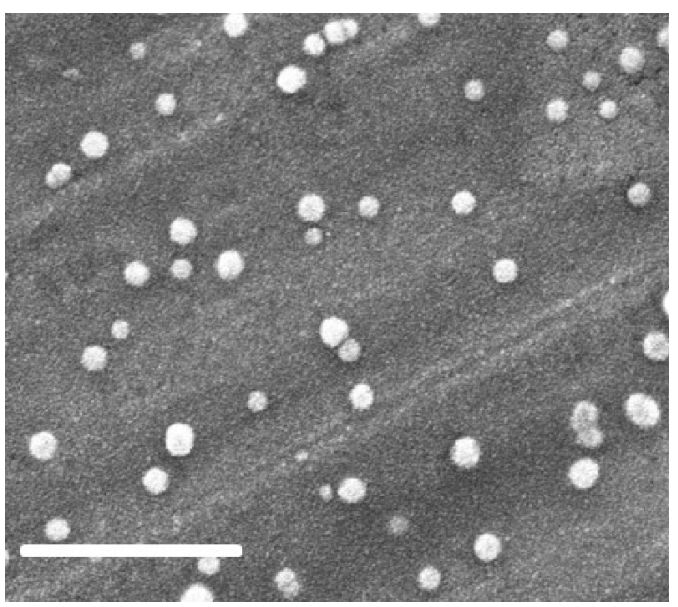

B

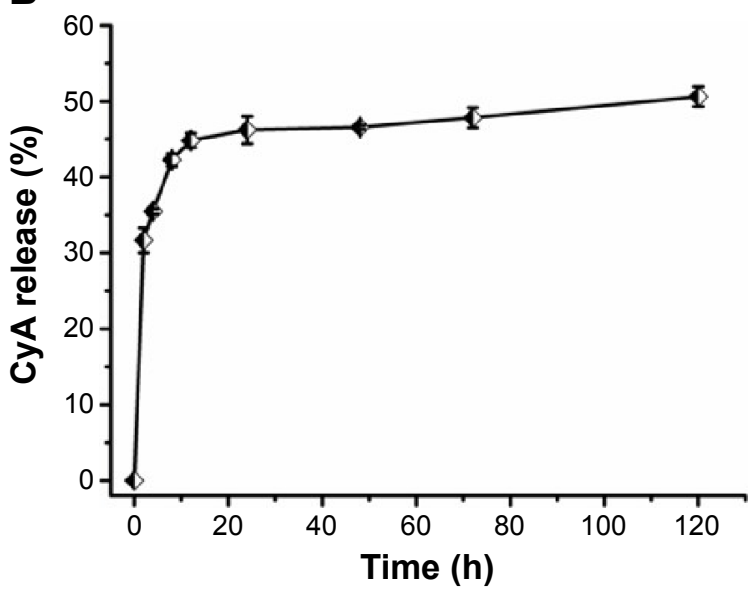

Figure 5 The morphology and drug release behavior of micelles.

Notes: (A) Morphology of polymeric micelles determined by scanning electron microscopy (SEM). Scale bar represents $200 \mathrm{~nm}$. (B) In vitro-release profile of CyA-loaded micelles at $\mathrm{pH} 7.2$.

Abbreviations: CyA, cyclosporine; h, hours.

in living C57BL/6 mice using two-photon scanning laser microscopy. ${ }^{29}$ We observed cross-sections of transparent cornea tissue using two-photon microscopy. The ocular distribution of the fluorescent model drug FDA was examined in mouse eyes after a topical instillation of the FDA-loaded micelle formulation.

When administrating the FDA-loaded polymeric micelle eye drops, deep corneal penetration of the dyes was observed (Figure 6), and the time dependence of the penetration was monitored. Over the first 15 minutes, the penetration of FDA was mostly limited to the epithelial layers of the cornea. However, a strong fluorescent signal corresponding to FDA that spread from the epithelium toward the stromal layers of the cornea was apparent at 30 minutes. By 60 minutes, the fluorescent signal in the stromal layers peaked and was stronger than that in the epithelial layers.

For the FDA nanoparticle and FDA microparticle control drops, fluorescent images were only taken of the superficial layer of the corneal epithelium (Figure 6B and C), and there were no obvious changes over time. For the

Table 2 Effect of initial drug content on its loading level in polymeric micelles, based on $10 \mathrm{mg}$ of polymer in $10 \mathrm{~mL}$ solution

\begin{tabular}{lll}
\hline $\begin{array}{l}\text { Initial drug } \\
\text { content (mg) }\end{array}$ & $\begin{array}{l}\text { Drug-loading } \\
\text { content (\%) }\end{array}$ & $\begin{array}{l}\text { Drug-loading } \\
\text { efficiency (\%) }\end{array}$ \\
\hline 2 & 8.32 & 13.60 \\
1 & 5.83 & 34.02 \\
0.75 & 4.63 & 69.68 \\
0.5 & 3.47 & 75.37 \\
0.2 & 1.55 & 80.05 \\
0.1 & 0.82 & 84.17 \\
\hline
\end{tabular}

FDA-loaded PEG-PCL micelle control drops, deep corneal penetration of the dye and its time-dependent penetration were monitored, but the FDA fluorescence signal was relatively weak.

\section{Discussion}

The eye has a complex structure and is highly resistant to foreign substances, including drugs. The opposing characteristics of the epithelium and the stroma prevent most drugs from effectively penetrating the cornea. Various efforts in ocular drug delivery have been made to prolong the residence time of drugs topically applied onto the eye and to improve their bioavailability. ${ }^{9}$ Many different colloidal delivery vehicles have been developed based on the potential use of polymeric nanoparticles as drug carriers. Among the reported colloidal delivery systems, polymeric micelles have promising and interesting characteristics for ophthalmic applications, such as their simple preparation and nanosize, the stability of their formulations, their transparency, their efficiency in drug loading, and their localized drug release. In addition, these micelles have several favorable biological properties, including biodegradability, mucoadhesiveness, biocompatibility, and nontoxicity.

In the present study, we successfully synthesized the triblock copolymer mPEG-PCL-g-PEI using ring-opening polymerization and an amidation reaction (Figure 1). Measurements made by ${ }^{1} \mathrm{H}$ NMR, IR, and GPC revealed the chemical structures of the copolymer (Figures 2 and 3).

As an amphiphilic diblock copolymer, the mPEG-PCL$g$-PEI copolymer self-assembled into polymeric micelles that 
A
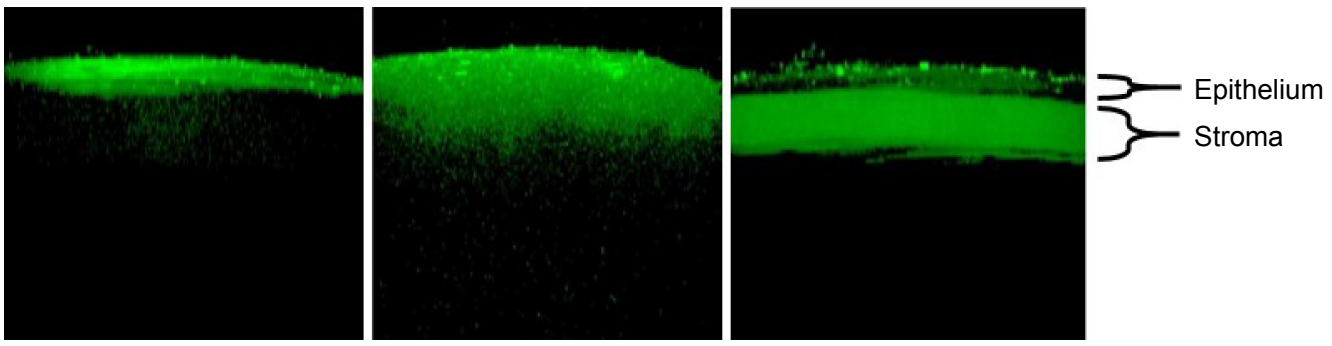

B
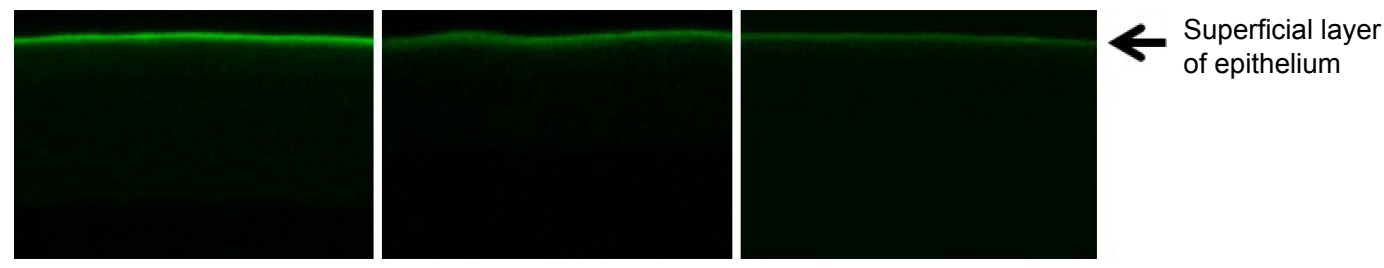

C
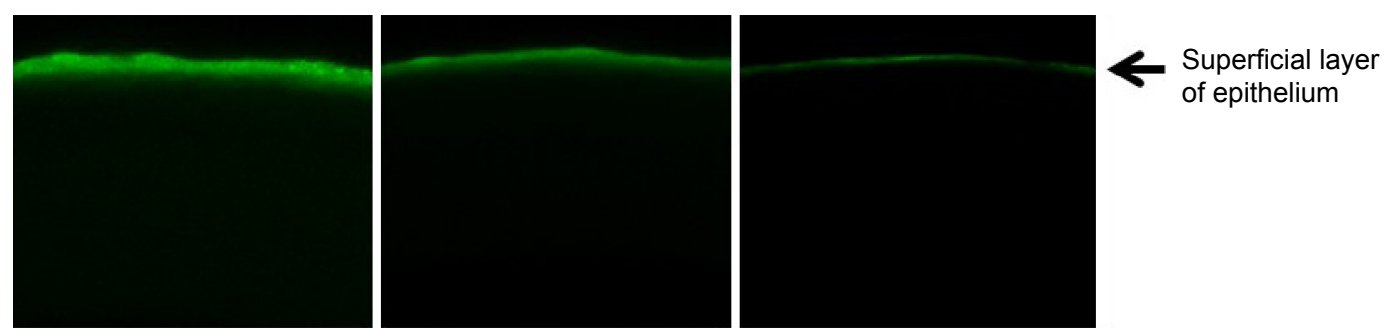

D

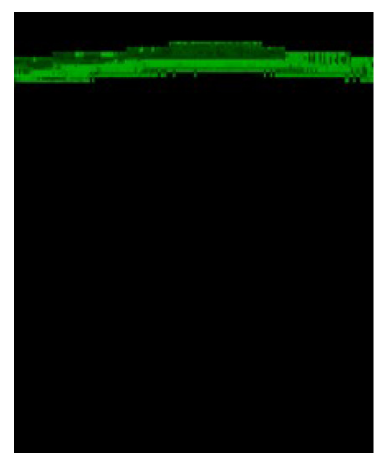

$15 \min$

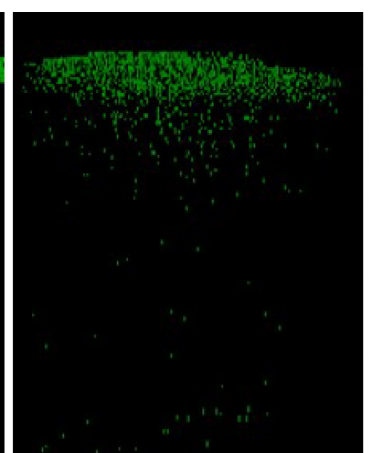

$30 \mathrm{~min}$

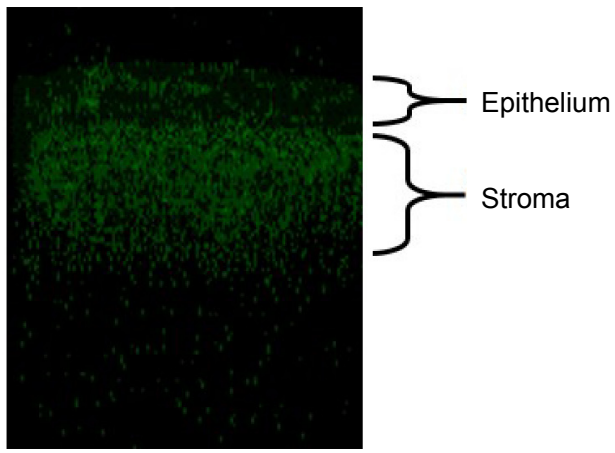

$60 \mathrm{~min}$

Time after administration of eye drops

Figure 6 Representative in vivo two-photon microscopy images of corneal cross-sections at I5 min, 30 min, and 60 min after administration of different types of eye drops in C57BL/6 mice.

Notes: The administered eye drops were (A) FDA-loaded PCl micelles, (B) FDA nanoparticles, (C) FDA microparticles, and (D) FDA-loaded PEG-PCL micelles (magnification, 100x).

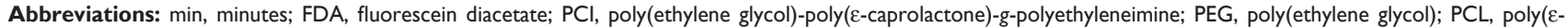
caprolactone).

were capable of delivering hydrophobic drugs. Each micelle contained PEG and PEI as a hydrophilic segment and PCL as a hydrophobic segment. The PEG chains on the micelle surfaces, which are hydrophilic, nontoxic, and nonimmunogenic, have been described as efficient drug carriers with a range of applications, including prolonging the systemic cycling time of drug-delivery systems. ${ }^{16,25}$ In addition, PEG has excellent solubility in many hydrophilic and hydrophobic solvents. As a hydrophobic polymer, a PCL block is biodegradable and is an ideal material for drug delivery and other 
applications. ${ }^{30}$ PEG-PCL micelles have been widely used as drug-delivery carriers to improve the solubility of hydrophobic drugs. ${ }^{17,31}$ In addition, PEI (Mw 1,800 Da) chains have been conjugated to classic PEG-PCL amphiphilic compounds to create superior cationic polymers due to their ability to develop molecular attraction forces via electrostatic interactions between the positively charged amino groups of PEI and the negatively charged sialic acid residues of mucin. There are also reports on the use of polymeric micelles prepared with mPEG-PCL- $g$-PEI copolymer for the codelivery of chemotherapy drugs and nucleic acid drugs into cancerous tumors. ${ }^{32}$ However, to date, there are no reports on the use of mPEG-PCL-g-PEI polymeric micelles for topical drug delivery in ocular applications.

Recent advancements in nanotechnology have encouraged researchers to find new ways of overcoming the corneal barrier. ${ }^{33}$ The particle size of a polymer nanoparticle is a key factor related to its ability to pass through biological membranes and has a significant effect on the rate of its ocular penetration in ophthalmic applications. Additionally, larger sized particles may lead to a scratching feeling based on foreign body sensation. ${ }^{9,34}$ Furthermore, the zeta potential of a polymeric micelle also affects the permeability of an encapsulated drug across the corneal barrier. Positively charged micelles enhance the permeability, presumably due to their binding of the negatively charged proteoglycan matrix, which prolongs the residence time of the drugs on the procornea. As studied previously, the use of cationic polymers can increase the transepithelial absorption of peptide drugs by causing the dissociation of tight junction assemblies, which restricts paracellular permeation. ${ }^{35}$

The polymeric micelles used in this study had a relatively small average hydrodynamic diameter of $27.74 \mathrm{~nm}$, a narrow size distribution with a PDI of 0.212 , and a moderate positive charge of $12.12 \mathrm{mV}$, all of which are consistent with previous reports (Table 1). ${ }^{23}$ Based on the SEM findings, all of the particles exhibited a spherical shape and were uniform in size (Figure 5A). Control groups were established to evaluate the effects of particle size (FDA microparticles group) and zeta potential (FDA nanoparticles group and PEG-PCL micelle group) on the corneal penetration of drugs.

Regarding in vitro drug release (Figure 5B), we found a burst release of $30 \%-40 \%$ in the initial 2 hours of observation; however, a slow, sustained release followed. This can be attributed to the extent of adsorption of the hydrophobic drug molecules to the polymeric micelles during the preparation of the drug-loaded micelles. Fast drug release from polymeric micelles can be attributed to the surface adsorption and faster drug diffusion away from the polymer at a $\mathrm{pH}$ of 7.2 , whereas slow drug release can be attributed to the deep loading of the drug into the polymeric micelle core, leading to a sustained release. The high DLC and biphasic-release mode enabled the polymeric micelles to deliver a high quantity of drug to the cornea, allowing an effective drug concentration to be quickly achieved, and the cornea thus became a drug depot that released the drug over time. In addition, with the micelle formulation, the number of instillations per day can be reduced, increasing patient compliance.

Two-photon laser scanning microscopy is a new technology that combines laser scanning microscopy with two-photon excitation and is suitable for the study of living cells and tissues, as well as for long-term observations. This method also enables in-depth imaging of thick biological samples. In the present study, two-photon laser scanning microscopy was adopted to perform in vivo 3-D imaging of the distribution of a fluorescent drug in mouse cornea. ${ }^{29,36}$

At present, there are numerous reports discussing the ocular penetration of drugs. ${ }^{8,9,18,37}$ To the best of our knowledge, this novel biometry method was used for the first time in vivo to clearly visualize the penetration behaviors of drugs into the corneas of $\mathrm{BALB} / \mathrm{c}$ mice.

As shown in Figure 6, a high degree of cornea penetration was observed after administering the PCI polymeric micelles, and the behavior was time dependent. Initially, the fluorescence signal was mainly concentrated in the epithelial layers of the cornea, but it eventually localized to the stromal layers. This study showed the ability of polymeric micelles to overcome the corneal barrier and to release an incorporated drug model into the corneal inner layer.

In contrast, no significant fluorescence was detected in the deep layers of the ocular cornea, (eg, the stroma), even 60 minutes after the administration of the FDA microparticle eye drops and FDA nanoparticle eye drops (Figure 6B and C). Although PEG-PCL micelles had a similar particle size, they had a neutral zeta potential and produced a relatively weak FDA fluorescence signal. This may be because nanomicelles with neutral or negatively charged do not possess a prolonged residence time on the ocular surface and do not facilitate cellular uptake. Although the concentrations of FDA were the same, these results indicate that the particle size and the zeta potential of the nanoparticles had a significant effect on the rate of the ocular penetration of the dye. The small size of the particles allows them to easily permeate through the tightly packed corneal epithelial cells and junctional complexes and to overcome the corneal barrier. Positively charged micelles 
can enhance corneal penetration by prolonging the ocular residence time and increasing the interaction with the negative cell membrane.

Furthermore, the PEGylated shells of the polymeric micelles may play a key role in enhancing the ocular penetration of micelle-encapsulated drugs. In addition to improving the stability of the micelles, PEG has outstanding solubility both in hydrophilic and hydrophobic solvents. ${ }^{25}$ This feature makes PEG-containing micelles even more effective in penetrating the amphipathic cornea, with its lipophilic epithelium and hydrophilic stroma, which create a strong permeation barrier to hydrophilic and lipophilic drugs in the eye. ${ }^{4}$ The polymeric micelles based on the triblock copolymer PCI that were created in this study can overcome the corneal barrier and effectively load and deliver drugs.

\section{Conclusion}

In this study, we synthesized and characterized the triblock copolymer PEG-PCL-g-PEI, which self-assembled into polymeric micelles. In addition, the self-assembled polymeric micelles were positively charged via their linkages to PEI. The drug-loaded micelles possessed small size $(28 \mathrm{~nm})$ and positive surface potential $(+12 \mathrm{mV})$. The results generated with FDA-labeled micelles in vivo and in vitro showed that the drug-loaded micelles markedly prolonged retention time and enhanced drug penetration in the cornea. Given its favorable biocompatibility and biodegradability, this well-defined ternary copolymer may have a promising role in ocular drug delivery. Detailed investigations of ocular penetration, the effects of particle size and polarity in polymeric micelles, and an understanding of the mechanisms by which polymeric micelles cross corneal barriers are currently underway.

\section{Acknowledgments}

This work was supported by the National Natural Science Foundation of China (grants 21504082, 81170831, 81270991, U1304811, and U1404832) and the Science and Technology projects of Zhengzhou Municipal Science and Technology Bureau (141PPTGG294 and 131PPTGG-378-3). The authors would like to thank Professor SM Zhang, Dr HM Zhang, and Dr ST Sun for their support of this study. We are grateful for the technical support of SS Liu and GM Chen.

\section{Disclosure}

The authors report no conflicts of interest in this work.

\section{References}

1. Ali Y, Lehmussaari K. Industrial perspective in ocular drug delivery. Adv Drug Deliv Rev. 2006;58(11):1258-1268.
2. Yang J, Yan J, Zhou Z, Amsden BG. Dithiol-PEG-PDLLA micelles: preparation and evaluation as potential topical ocular delivery vehicle. Biomacromolecules. 2014;15(4):1346-1354.

3. Shirasaki Y. Molecular design for enhancement of ocular penetration. J Pharm Sci. 2008;97(7):2462-2496.

4. Gaudana R, Ananthula HK, Parenky A, Mitra AK. Ocular drug delivery. AAPS J. 2010;12(3):348-360.

5. Mishima S. Clinical pharmacokinetics of the eye. Proctor lecture. Invest Ophthalmol Vis Sci. 1981;21(4):504-541.

6. Gaudana R, Jwala J, Boddu SHS, Mitra AK. Recent perspectives in ocular drug delivery. Pharm Res. 2009;26(5):1197-1216.

7. Kompella UB, Amrite AC, Ravi RP, Durazo SA. Nanomedicines for back of the eye drug delivery, gene delivery, and imaging. Prog Retin Eye Res. 2013;36(9):172-198.

8. Vaishya RD, Gokulgandhi M, Patel S, Minocha M, Mitra AK. Novel dexamethasone-loaded nanomicelles for the intermediate and posterior segment uveitis. AAPS Pharm Sci Tech. 2014;15(5):1238-1251.

9. Baba K, Tanaka Y, Kubota A, et al. A method for enhancing the ocular penetration of eye drops using nanoparticles of hydrolyzable dye. J Control Release. 2011;153(3):278-287.

10. Araujo J, Gonzalez E, Egea MA, Garcia ML, Souto EB. Nanomedicines for ocular NSAIDs: safety on drug delivery. Nanomedicine. 2005;5(4): 394-401.

11. Bu HZ, Gukasyan HJ, Goulet L, Lou XJ, Xiang C, Koudriakova T. Ocular disposition, pharmacokinetics, efficacy and safety of nanoparticle-formulated ophthalmic drugs. Curr Drug Metab. 2007; 8(2):91-107.

12. Mannermaa E, Vellonen KS, Urtti A. Drug transport in corneal epithelium and blood-retina barrier: emerging role of transporters in ocular pharmacokinetics. Adv Drug Deliv Rev. 2006;58(11):1136-1163.

13. Miyata K, Christie RJ, Kataoka K. Polymeric micelles for nano-scale drug delivery. React Funct Polym. 2011;71(3):227-234.

14. Owen SC, Chan DPY, Shoichet MS. Polymeric micelle stability. Nano Today. 2012;7(1):53-65.

15. Kwon GS, Kataoka K. Block copolymer micelles as long-circulating drug vehicles. Adv Drug Deliv Rev. 2012;64:295-309.

16. Li J, Yu X, Wang Y, et al. A reduction and $\mathrm{pH}$ dual-sensitive polymeric vector for long-circulating and tumor-targeted siRNA delivery. Adv Mater. 2014;26(48):8217-8224.

17. Li Z, Wu X, Li J, et al. Antitumor activity of celastrol nanoparticles in a xenograft retinoblastoma tumor model. Int J Nanomedicine. 2012;7: 2389-2398.

18. Chopra P, Hao J, Li SK. Sustained release micellar carrier systems for iontophoretic transport of dexamethasone across human sclera. $J$ Control Release. 2012;160(1):96-104.

19. Vaishya RD, Khurana V, Patel S, Mitra AK. Controlled ocular drug delivery with nanomicelles. Wiley Interdiscip Rev Nanomed Nanobiotechnol. 2014;6(5):422-437.

20. Civiale C, Licciardi M, Cavallaro G, Giammonab G, Mazzone MG. Polyhydroxyethylaspartamide-based micelles for ocular drug delivery. Int J Pharm. 2009;378(378):177-186.

21. Mourad F. OChiPEG micelles proven to facilitate the transscleral delivery of rapamycin. Invest Ophthalmol Vis Sci. 2012;53(13):8138.

22. Nagarwal RC, Kant S, Singh PN, Maiti P, Pandit JK. Polymeric nanoparticulate system: a potential approach for ocular drug delivery. J Control Release. 2009;136(1):2-13.

23. Endres TK, Beck-Broichsitter M, Samsonova O, Renette T, Kissel TH. Self-assembled biodegradable amphiphilic PEG-PCL-IPEI triblock copolymers at the borderline between micelles and nanoparticles designed for drug and gene delivery. Biomaterials. 2011;32(30):7721-7731.

24. Shuai X, Merdan T, Unger F, Wittmar M, Kissel T. Novel biodegradable ternary copolymers hy-PEI-g-PCL-b-PEG: synthesis, characterization, and potential as efficient nonviral gene delivery vectors. Macromolecules. 2003;36(15):5751-5759.

25. Li J, Cheng D, Yin T, et al. Copolymer of poly(ethylene glycol) and poly(L-lysine) grafting polyethylenimine through a reducible disulfide linkage for siRNA delivery. Nanoscale. 2014;6(3):1732-1740. 
26. Chidambaram N, Burgess DJ. A novel in vitro release method for submicron sized dispersed systems. AAPS Pharm Sci. 1999;1(3):32-40.

27. Liu Y, Nguyen J, Steele T, Merkel O, Kissel T. A new synthesis method and degradation of hyper-branched polyethylenimine grafted polycaprolactone block mono-methoxyl poly (ethylene glycol) copolymers (hy-PEI-gPCL-b-mPEG) as potential DNA delivery vectors. Polymer. 2009;50(16): 3895-3904.

28. Shi S, Fan M, Wang X, et al. Synthesis and characterization of mPEGPCL-g-PEI and self-assembled nanoparticle uptake in vitro and in vivo. J Phys Chem C. 2010;114(49):21315-21321.

29. Zhang H, Wang L, Xie Y, et al. The measurement of corneal thickness from center to limbus in vivo in $\mathrm{C} 57 \mathrm{BL} / 6$ and $\mathrm{BALB} / \mathrm{c}$ mice using two-photon imaging. Exp Eye Res. 2013;115:255-262.

30. Loh XJ, Colin SKB, Li J. Synthesis and water-swelling of thermoresponsive poly (ester urethane) s containing poly ( $\varepsilon$-caprolactone), poly (ethylene glycol) and poly (propylene glycol). Biomaterials. 2008; 29(22):3185-3194.

31. Li Z, Yao L, Li J, et al. Celastrol nanoparticles inhibit corneal neovascularization induced by suturing in rats. Int J Nanomedicine. 2012;7: 1163-1173.
32. Shi S, Shi K, Tan L, et al. The use of cationic MPEG-PCL-g-PEI micelles for co-delivery of Msurvivin T34A gene and doxorubicin. Biomaterials. 2014;35(15):4536-4547.

33. Di Tommaso C, Bourges JL, Valamanesh F, et al. Novel micelle carriers for cyclosporin A topical ocular delivery: in vivo cornea penetration, ocular distribution and efficacy studies. Eur J Pharm Biopharm. 2012;81(2): 257-264.

34. Zimmer A, Kreuter J. Microspheres and nanoparticles used in ocular delivery systems. Adv Drug Deliv Rev. 1995;16(1):61-73.

35. Nemoto E, Ueda H, Akimoto M, Natsume H, Morimoto Y. Ability of poly-L-arginine to enhance drug absorption into aqueous humor and vitreous body after instillation in rabbits. Biol Pharm Bull. 2007;30(9): 1768-1772.

36. Zhang H, Wang L, Liu S, et al. Two-photon imaging of the cornea visualized in the living mouse using vital dyes. Invest Ophthalmol Vis Sci. 2013;54(10):6526-6536.

37. Sosnová-Netuková M, Kuchynka P, Forrester JV. The suprabasal layer of corneal epithelial cells represents the major barrier site to the passive movement of small molecules and trafficking leukocytes. $\mathrm{Br} J$ Ophthalmol. 2007;91(3):372-378.
International Journal of Nanomedicine

\section{Publish your work in this journal}

The International Journal of Nanomedicine is an international, peerreviewed journal focusing on the application of nanotechnology in diagnostics, therapeutics, and drug delivery systems throughout the biomedical field. This journal is indexed on PubMed Central, MedLine, CAS, SciSearch $\AA$, Current Contents $₫ /$ Clinical Medicine,

\section{Dovepress}

Journal Citation Reports/Science Edition, EMBase, Scopus and the Elsevier Bibliographic databases. The manuscript management system is completely online and includes a very quick and fair peer-review system, which is all easy to use. Visit http://www.dovepress.com/ testimonials.php to read real quotes from published authors. 